\title{
A Method on Solving Multiobjective Conditional Value-at-Risk ${ }^{\star}$
}

\author{
Min Jiang ${ }^{1}$, Qiying $\mathrm{Hu}^{2}$, and Zhiqing Meng ${ }^{3}$ \\ 1 School of Economics and Management, \\ Xidian University, Xi'an, 710071, China \\ j_yx@263.net \\ 2 College of International Business \& Management, \\ Shanghai University, Shanghai, 201800,China \\ huqiying@sina.com \\ 3 College of Business and Administration, \\ Zhejiang University of Technology, Hangzhou, 310032, China \\ mengzhiqing@xtu.edu.cn
}

\begin{abstract}
This paper studies Conditional Value-at-Risk (CVaR) with multiple losses. We introduce the concept of $\boldsymbol{\alpha}$-CVaR for the case of multiple losses under the confidence level vector $\boldsymbol{\alpha}$. The $\boldsymbol{\alpha}$-CVaR indicates the conditional expected losses corresponding to the $\boldsymbol{\alpha}$-VaR. The problem of solving the minimal $\boldsymbol{\alpha}$-CVaR results in a multiobjective problem (MCVaR). In order to get Pareto efficient solutions of the (MCVaR), we introduce a single objective problem (SCVaR) and show that the optimal solutions of the (SCVaR) are the Pareto efficient solutions of (MCVaR).
\end{abstract}

Keywords: Credit risk, Loss functions, $\boldsymbol{\alpha}$-CVaR, Pareto efficient solutions

\section{Introduction}

Value-at-Risk (VaR) is a measure for the potential loss in the financial market. With respect to a specified probability level $\alpha$, the $\alpha$-VaR of a portfolio is the lowest amount $y$ such that, with probability $\alpha$, the loss will not exceed $y$. VaR has achieved its great success in practice. However, research shows that VaR has undesirable properties both in theory and in practice. The main undesirable characteristics are as follows [1]. (a) There are various methodologies for modeling VaR, such as Historical Simulation, Monte Carlo, Extreme Value Theory, but the results using these methodologies are discrepant. (b) It is difficult to solve directly the problem of optimizing VaR. (c) VaR dissatisfies sub-additivity, which leads that financial organizations cannot obtain the whole VaR from those of their branches. (d) It is computed the loss exceeding VaR with likehood $(1-\alpha)$,

* The project was supported by the National Natural Science Foundation of China with grant 72072021. 
but no information is provided on the amount of the excess loss, which may be significantly greater.

The concept of Conditional Value at Risk (CVaR) ([2]) was presented mainly to solve the problem of sub-additivity. With a specified probability $\alpha$, the $\alpha$ CVaR is the conditional expectation of losses above the VaR. CVaR overcomes several limitations of VaR and has good properties, especially its good computability. Recently, there are several studies on CVaR area. Chernozhukov and Umantsev in [3] stressed the important aspects of measuring the external and intermediate conditional risk and gave an empirical application characterizing the key economic determinants of various levels of conditional risk. Andersson, Mausser, Rosen et al. in [4] presented an efficient algorithm to minimize CVaR. Rockafellar and Uryasev [5] derived some fundamental properties of conditional value-at-risk (CVaR) for loss distributions in Finance that can involve discreetness.

However, the losses that should be considered in practical risk management are often multiple, such as those due to interest risk, exchange risk, shares risk, commercial risk. So, the risk management problems are of multiobjective. Krokhmal, Palmquist and Uryasev [6] and Wang and Li [7] solve efficient frontier problems with three losses under the framework of CVaR. However, they did not concern in theoretical studies.

This paper will study the multiple conditional VaR. After introducing the concept of $\boldsymbol{\alpha}$-VaR with confidence level vector $\boldsymbol{\alpha}$ of a portfolio for multi losses based on the paper [2], we define $\boldsymbol{\alpha}$-CVaR as the conditional expected losses above the $\boldsymbol{\alpha}$-VaR. We then present the optimization problem of minimizing the multiple $\boldsymbol{\alpha}$-CVaR as a multiple programming. We prove that under some conditions the multiple $\boldsymbol{\alpha}$-CVaR problem can be transformed into a single objective nonlinear programming problem, which can be solved relative simply.

The remainder of this paper is organized as follows. In Section 2, we introduce the concept of $\alpha-\mathrm{CVaR}$ and the main results of paper [2]. Then, in Section 3, we define the multiple $\boldsymbol{\alpha}$-CVaR. In Section 4, we show the main results of solving the multiple $\boldsymbol{\alpha}$-CVaR. Section 5 gives the conclusions.

\section{CVaR with Single Loss}

Rockfellar and Uryasev [2] discussed the CVaR model with single loss. Their main concepts and results are as follows.

Let $\boldsymbol{x}=\left(x_{1}, x_{2}, \ldots, x_{n}\right)^{T}$ be the portfolio, which is also called the decision vector, with $x \in X$ and $X \subset R^{n}$. Let $f(x, \xi): R^{n} \times R^{m} \rightarrow R^{1}$ be the loss associated with the decision vector $\boldsymbol{x}$, where $\boldsymbol{\xi}=\left(\xi_{1}, \xi_{2}, \ldots, \xi_{m}\right)^{T}$ is a random vector representing the uncertainties that affect the loss. For simplicity, it is assumed that $\xi$ is a continuously random variable with p.d.f. $p(z)$. The cumulative distribution function of $f(\boldsymbol{x}, \xi)$ is denoted by

$$
\Psi(\boldsymbol{x}, y)=P\{f(\boldsymbol{x}, \xi) \leq y\}=\int_{f(\boldsymbol{x}, z) \leq y} p(z) d z,
$$

which is nondecreasing and right continuous in $y$ for each $\boldsymbol{x} \in X$. 
Definition 2.1 Given $\alpha \in(0,1)$ and $\boldsymbol{x} \in X$, the $\alpha$-VaR (of the loss) associated with the decision $\boldsymbol{x}$ under the confidence level $\alpha$ is defined by

$$
y_{\alpha}(\boldsymbol{x})=\min \{y \in R: \Psi(\boldsymbol{x}, y) \geq \alpha\}
$$

which is the lowest amount $y$ such that, with probability $\alpha$, the loss will not exceed $y$. Moreover, $\alpha$-CVaR (of the loss) associated with $\boldsymbol{x}$ under $\alpha$ is defined by

$$
\phi_{\alpha}(\boldsymbol{x})=(1-\alpha)^{-1} \int_{f(\boldsymbol{x}, z) \geq y_{\alpha}(\boldsymbol{x})} f(\boldsymbol{x}, z) p(z) d z .
$$

$\phi_{\alpha}(\boldsymbol{x})$ is the conditional expectation of the loss associated with $\boldsymbol{x}$ relatively to that loss being the $\alpha$-VaR $y_{\alpha}(\boldsymbol{x})$ or greater. It is difficult to work directly with the $\alpha$-CVaR because of unknowing $y_{\alpha}(\boldsymbol{x})$. Rockfellar and Uryasev introduced a simpler function:

$$
F_{\alpha}(\boldsymbol{x}, y)=y+(1-\alpha)^{-1} \int_{z \in R}[f(\boldsymbol{x}, z)-y]^{+} p(z) d z
$$

where $t^{+}=\max (0, t)$.

Lemma 2.1 As a function of y, $F_{\alpha}(\boldsymbol{x}, y)$ is convex, continuously differentiable and

$$
\frac{\partial}{\partial y} F_{\alpha}(\boldsymbol{x}, y)=(1-\alpha)^{-1}\left[\Psi_{\alpha}(\boldsymbol{x}, y)-\alpha\right] .
$$

Under the condition of

$$
P\{f(\boldsymbol{x}, \xi)=y\}=\int_{f(\boldsymbol{x}, z)=y} p(z) d z=0,
$$

the following result is shown.

Theorem 2.1 Minimizing the $\alpha$-CVaR $\phi_{\alpha}(\boldsymbol{x})$ over $\boldsymbol{x} \in X$ is equivalent to minimizing $F_{\alpha}(\boldsymbol{x}, y)$ over $(\boldsymbol{x}, y) \in X \times R$, in the sense that

$$
\min _{\boldsymbol{x} \in X} \phi_{\alpha}(\boldsymbol{x})=\min _{(\boldsymbol{x}, y) \in X \times R} F_{\alpha}(\boldsymbol{x}, y) .
$$

Furthermore, $F_{\alpha}(\boldsymbol{x}, y)$ is convex with respect to $(\boldsymbol{x}, y)$ and $\phi_{\alpha}(\boldsymbol{x})$ is convex with respect to $\boldsymbol{x}$ when $f(\boldsymbol{x}, y)$ is convex with respect to $\boldsymbol{x}$. In this case, if $X$ is a convex set, the above joint minimization is a convex programming.

According to Theorem 2.1, for the purpose of determining an $\boldsymbol{x}$ that yields the minimum $\alpha$-CVaR, it is not necessary to minimize directly the function $\phi_{\alpha}(\boldsymbol{x})$ which may be hard to do. Instead, one can operate on the far simpler function $F_{\alpha}(\boldsymbol{x}, y)$.

Theorem 2.1 opens the door to minimize $\alpha$-CVaR over $\boldsymbol{x} \in X$ and so we can use it to solve practical risk management problems. 


\section{$3 \quad$ CVaR with Multiple Losses}

In this section, we generalize the concepts of $\alpha$-VaR and $\alpha$-CVaR into the case of multiple losses.

Let $f_{i}(\boldsymbol{x}, \boldsymbol{\xi}) \in R^{n} \times R^{m} \rightarrow R^{1}(i=1,2, \cdots, I)$ be the loss functions which depends upon the decision vector $\boldsymbol{x} \in X \subset R^{n}$ and the random vector $\boldsymbol{\xi} \in R^{m}$. $X$ is the set of possible portfolios. For simplicity, we assume that $\xi$ is a random variable with the probability density function $p(z)$. However, this assumption is not critical for our discussions in the following. Denote by $\Psi_{i}(\boldsymbol{x}, \cdot)$ the distribution function of the loss $f_{i}(\boldsymbol{x}, \xi)$, i.e.,

$$
\Psi_{i}(\boldsymbol{x}, y)=P\left\{f_{i}(\boldsymbol{x}, \xi) \leq y\right\}=\int_{f_{i}(\boldsymbol{x}, z) \leq y} p(z) d z, \quad i=1,2, \cdots, I .
$$

We give the following definition.

Definition 3.1 Given $\alpha_{i} \in(0,1), i=1,2, \cdots, I$, and $\boldsymbol{x} \in X$, the $\boldsymbol{\alpha}$-VaR (of the loss) associated with $\boldsymbol{x}$ under the confidence level vector $\boldsymbol{\alpha}=\left(\alpha_{1}, \alpha_{2}, \cdots, \alpha_{I}\right)$ is defined by

$$
y^{*}(\boldsymbol{x})=\min \left\{y \mid \Psi_{i}(\boldsymbol{x}, y) \geq \alpha_{i}, \quad i=1,2, \cdots, I\right\} .
$$

When $I=1$, the $\boldsymbol{\alpha}$-VaR is exactly the $\alpha$-VaR for one loss defined by Definition 2.1. For $i=1,2, \cdots, I, \Psi_{i}(\boldsymbol{x}, y) \geq \alpha_{i}$, or $P\left\{f_{i}(\boldsymbol{x}, \xi) \leq y\right\} \geq \alpha_{i}$, means that, with probability $\alpha_{i}$, the $i$ th loss will not exceed $y$ for the portfolio $\boldsymbol{x}$. So,

$$
y_{i}^{*}(\boldsymbol{x})=\min \left\{y \mid \Psi_{i}(\boldsymbol{x}, y) \geq \alpha_{i}\right\}
$$

is the $\alpha_{i}$-VaR of $x$ for the $i$ th loss. Therefore, the $\boldsymbol{\alpha}$-VaR $y^{*}(x)$ for a portfolio $\boldsymbol{x}$ is the lowest amount $y$ such that $i$ th loss will not exceed $y$ with probability $\alpha_{i}$ for each $i$. Clearly, when $\Psi_{i}(\boldsymbol{x}, y)$ is nondecreasing and continuous in $y, y_{i}^{*}(\boldsymbol{x})$ is the smallest root of the equation $\Psi_{i}(\boldsymbol{x}, y)=\alpha_{i}$.

Obviously, given the confidence level vector $\boldsymbol{\alpha}=\left(\alpha_{1}, \alpha_{2}, \cdots, \alpha_{I}\right), y^{*}(\boldsymbol{x})$ is the maximum of the $\alpha_{i}$-VaR, i.e., $y^{*}(\boldsymbol{x})=\max \left\{y_{1}^{*}(\boldsymbol{x}), y_{2}^{*}(\boldsymbol{x}), \cdots, y_{I}^{*}(\boldsymbol{x})\right\}$.

When we discuss $\boldsymbol{\alpha}$-VaR of $\boldsymbol{x}$, we want to know how much the expected losses of $\mathrm{VaR}$ is. For each $i=1,2, \cdots, I$, we define

$$
\phi_{\alpha_{i}}(\boldsymbol{x}, y)=\left(1-\alpha_{i}\right)^{-1} \int_{f_{i}(\boldsymbol{x}, z) \geq y} f_{i}(\boldsymbol{x}, z) p(z) d z .
$$

Then, we have

$$
\phi_{\alpha_{i}}(\boldsymbol{x}, y)=\left(1-\alpha_{i}\right)^{-1} P\left\{f_{i}(\boldsymbol{x}, \xi) \geq y\right\} E\left\{f_{i}(\boldsymbol{x}, \xi) \mid f_{i}(\boldsymbol{x}, \xi) \geq y\right\} .
$$

When $y=y_{i}^{*}(\boldsymbol{x}), P\left\{f_{i}(\boldsymbol{x}, \xi) \geq y_{i}^{*}(\boldsymbol{x})\right\}=\alpha_{i}$ and so $\phi_{\alpha_{i}}\left(\boldsymbol{x}, y_{i}^{*}(\boldsymbol{x})\right)=E\left\{f_{i}(\boldsymbol{x}, \xi)\right.$ $\left.f_{i}(\boldsymbol{x}, \xi) \geq y_{i}^{*}(\boldsymbol{x})\right\}$ is exactly the conditional expected $i$ th loss for the portfolio $\boldsymbol{x}$. Thus we use $\phi_{\alpha_{i}}\left(\boldsymbol{x}, y^{*}(\boldsymbol{x})\right)$ to approximate the conditional expected $i$ th loss for $\boldsymbol{x}$ and $\alpha_{i}$ under the loss $y^{*}(\boldsymbol{x})$. It describes the risk of the portfolio $\boldsymbol{x}$ for the $i$ th loss. If $\phi_{\alpha_{i}}\left(\boldsymbol{x}, y^{*}(\boldsymbol{x})\right)$ is small, the risk will be also small. 
Hence, $\left(\phi_{\alpha_{1}}\left(\boldsymbol{x}, y^{*}(\boldsymbol{x})\right), \phi_{\alpha_{2}}\left(\boldsymbol{x}, y^{*}(\boldsymbol{x})\right), \cdots, \phi_{\alpha_{I}}\left(\boldsymbol{x}, y^{*}(\boldsymbol{x})\right)\right)$ describes the conditional expected losses under the $\boldsymbol{\alpha}$-VaR $y^{*}(\boldsymbol{x})$ for the portfolio $\boldsymbol{x}$ and the confidence level vector $\boldsymbol{\alpha}$.

Definition 3.2 For given $\alpha_{i} \in(0,1), i=1,2, \cdots, I$, and $\boldsymbol{x} \in X$, we call the vector $\left(\phi_{\alpha_{1}}\left(\boldsymbol{x}, y^{*}(\boldsymbol{x})\right), \phi_{\alpha_{2}}\left(\mathbf{x}, y^{*}(\boldsymbol{x})\right), \cdots, \phi_{\alpha_{I}}\left(\boldsymbol{x}, y^{*}(\boldsymbol{x})\right)\right)$ the $\boldsymbol{\alpha}$-CVaR associated with $\boldsymbol{x}$ under the confidence level vector $\boldsymbol{\alpha}=\left(\alpha_{1}, \cdots, \alpha_{I}\right)$.

It is not easy to compute $\phi_{\alpha_{i}}(\boldsymbol{x}, y)$. Thus we introduce another loss function as in [2]. For $i=1,2, \cdots, I$, we define

$$
F_{\alpha_{i}}(\boldsymbol{x}, y)=y+\left(1-\alpha_{i}\right)^{-1} \int_{z \in R}\left[f_{i}(\boldsymbol{x}, z)-y\right]^{+} p(z) d z .
$$

By Lemma 2.1, we have the following result under the condition that all the loss functions satisfy (4), i.e., $P\left\{f_{i}(x, \xi)=y\right\}=0$ for $i=1,2, \cdots, I$.

Lemma 3.1 For each $i=1,2, \cdots, I, F_{\alpha_{i}}(\boldsymbol{x}, y)$ is a continuous differential and convex function, and

$$
\begin{aligned}
& \min _{y \in R} F_{\alpha_{i}}(\boldsymbol{x}, y)=\phi_{\alpha_{i}}\left(\boldsymbol{x}, y_{i}^{*}(\boldsymbol{x})\right) \\
& \frac{\partial F_{\alpha_{i}}(\boldsymbol{x}, y)}{\partial y}=\left(1-\alpha_{i}\right)^{-1}\left[\Psi_{\alpha_{i}}(\mathbf{x}, y)-\alpha_{i}\right]
\end{aligned}
$$

\section{Main Results}

We need to consider the minimal $\boldsymbol{\alpha}$-CVaR of $\boldsymbol{x} \in X$. That is, we face the following multiobjective problem:

$$
\begin{aligned}
&(\mathrm{MCVaR}) \min \left(\phi_{\alpha_{1}}\left(\boldsymbol{x}, y^{*}(\boldsymbol{x})\right), \phi_{\alpha_{2}}\left(\boldsymbol{x}, y^{*}(\boldsymbol{x})\right), \cdots, \phi_{\alpha_{I}}\left(\boldsymbol{x}, y^{*}(\boldsymbol{x})\right)\right) \\
& \text { s. t. } \boldsymbol{x} \in X .
\end{aligned}
$$

We first introduce the concept of efficient solutions in mutliobjective problems. Consider a multiobjective problem ([8])

$$
\begin{aligned}
& (\mathrm{MP}) \min \left(h_{1}(y), h_{2}(y), \cdots, h_{I}(y)\right) \\
& \text { s. t. } y \in Y,
\end{aligned}
$$

with $h_{i}: R^{m} \rightarrow R, 1=1,2, \cdots, I$ and $Y \subset R^{m}$. For $y^{*} \in Y$, if there is no $y \in Y$ such that

$$
h_{1}(y) \leq h_{1}\left(y^{*}\right), h_{2}(y) \leq h_{2}\left(y^{*}\right), \cdots, h_{I}(y) \leq h_{I}\left(y^{*}\right)
$$

where at least one inequality is strict, then $y^{*}$ is called a Pareto efficient solution to $(\mathrm{MP})$.

For the given $\boldsymbol{\alpha}=\left(\alpha_{1}, \alpha_{2}, \cdots, \alpha_{I}\right)$, if $\boldsymbol{x}^{*}$ is a Pareto efficient solution to (MCVaR), then $\left(\phi_{\alpha_{1}}\left(\boldsymbol{x}^{*}, y^{*}\left(\boldsymbol{x}^{*}\right)\right), \phi_{\alpha_{2}}\left(\boldsymbol{x}^{*}, y^{*}\left(\boldsymbol{x}^{*}\right)\right), \cdots, \phi_{\alpha_{I}}\left(\boldsymbol{x}^{*}, y^{*}\left(\boldsymbol{x}^{*}\right)\right)\right)$ is called 
a Pareto- $\boldsymbol{\alpha}$-CVaR and $\boldsymbol{x}^{*}$ is called a Pareto- $\boldsymbol{\alpha}$-CVaR efficient solution. The set of all Pareto- $\boldsymbol{\alpha}$-CVaR efficient solutions is denoted by $E(\boldsymbol{\alpha})$. In the following, we want to find out such an efficient solution.

It is difficult to compute $\phi_{\alpha_{i}}\left(\boldsymbol{x}, y^{*}(\boldsymbol{x})\right)$ from (7), so do (MCVaR). Therefore, we should consider other problems. First, we have the following lemma.

Lemma 4.1 For each $\boldsymbol{x} \in X$ and $y \in R$, we have that

$$
\phi_{\alpha_{i}}\left(\boldsymbol{x}, y^{*}(\boldsymbol{x})\right) \leq F_{\alpha_{i}}(\boldsymbol{x}, y), \quad i=1,2, \cdots, I .
$$

Proof. Since that $\phi_{\alpha_{i}}(\boldsymbol{x}, y)$ is nonincreasing in $y$, we have that

$$
\phi_{\alpha_{i}}\left(\boldsymbol{x}, y^{*}(\boldsymbol{x})\right) \leq \phi_{\alpha_{i}}\left(\boldsymbol{x}, y_{i}^{*}(\boldsymbol{x})\right) .
$$

Then by Lemma 3.1, we obtain (11).

For the given weight $0<\lambda_{i}<1, i=1,2, \cdots, I$, we consider the problem $\min _{y \in R} \sum_{i=1}^{I} \lambda_{i} F_{\alpha_{i}}(\boldsymbol{x}, y)$, for which we have the following theorem.

Theorem 4.1 Let $\boldsymbol{x} \in X$. Suppose that $\bar{y}$ is an optimal solution to the problem $\min _{y \in R} \sum_{i=1}^{I} \lambda_{i} F_{\alpha_{i}}(\boldsymbol{x}, y)$ and satisfies the condition

$$
P\left\{f_{i}(\boldsymbol{x}, \xi)=\bar{y}\right\}=0, \quad i=1,2, \cdots, I .
$$

Then

$$
\sum_{i=1}^{I} \lambda_{i} \phi_{\alpha_{i}}(\boldsymbol{x}, \bar{y})=\min _{y \in R} \sum_{i=1}^{I} \lambda_{i} F_{\alpha_{i}}(\boldsymbol{x}, y) .
$$

Proof. Let $F(\boldsymbol{x}, y)=\sum_{i=1}^{I} \lambda_{i} F_{\alpha_{i}}(\boldsymbol{x}, y)$. By Lemma 3.1, we have that

$$
\sum_{i=1}^{I} \lambda_{i}\left(1-\alpha_{i}\right)^{-1}\left(\Psi_{i}(\boldsymbol{x}, \bar{y})-\alpha_{i}\right)=0 .
$$

With this and (7), we have

$$
\sum_{i=1}^{I} \lambda_{i} F_{\alpha_{i}}(\boldsymbol{x}, \bar{y})=\sum_{i=1}^{I} \lambda_{i} \phi_{\alpha_{i}}(\boldsymbol{x}, \bar{y})+\bar{y} \sum_{i=1}^{I} \lambda_{i}\left(1-\alpha_{i}\right)^{-1}\left(\Psi_{i}(\mathbf{x}, \bar{y})-\alpha_{i}\right),
$$

which together with (15) implies (14).

When $f_{i}(\boldsymbol{x}, \xi)$ is a continuous random variable, the condition (13) must be true. But, when $f_{i}(\boldsymbol{x}, \xi)$ is a discrete type random variable, (13) holds if and only if that $\bar{y}$ is not in the range of $f_{i}(\boldsymbol{x}, \xi)$.

Now, suppose that $\Psi_{i}(\boldsymbol{x}, y)$ is strictly increasing in $y$. By Lemma 4.1 and Theorem 4.1, we have the following results.

Corollary 4.1 Under the conditions given in Theorem 4.1, $\bar{y}=y^{*}(\boldsymbol{x})$ if and only if

$$
\Psi_{i}(\boldsymbol{x}, \bar{y})-\alpha_{i}=0, \quad i=1,2, \cdots, I .
$$


Corollary 4.2 For any $\boldsymbol{x} \in X$,

$$
\sum_{i=1}^{I} \lambda_{i} \phi_{\alpha_{i}}\left(\boldsymbol{x}, y^{*}(\boldsymbol{x})\right) \leq \min _{y \in R} \sum_{i=1}^{I} \lambda_{i} F_{\alpha_{i}}(\boldsymbol{x}, y) .
$$

Moreover, if the problem $\min _{y \in R} \sum_{i=1}^{I} \lambda_{i} F_{\alpha_{i}}(\boldsymbol{x}, y)$ has an optimal solution $\bar{y}$ that satisfies (13) and (16), then the inequality (17) becomes an eqality.

Based on the above discussions, we now consider the following single objective problem.

$$
\begin{aligned}
& \min \sum_{i=1}^{I} \lambda_{i} F_{\alpha_{i}}(\boldsymbol{x}, y), \\
& \text { s. t. } y \in R, \boldsymbol{x} \in X .
\end{aligned}
$$

We have the following theorem to relate this single objective problem to the multiobjective problem (MCVaR).

Theorem 4.2 If $(\overline{\boldsymbol{x}}, \bar{y})$ is an optimal solution to (18) and $\bar{y}$ satisfies (13) and (16) for $\boldsymbol{x}=\overline{\boldsymbol{x}}$, then $\overline{\boldsymbol{x}}$ is a Pareto- $\alpha$-CVaR efficient solution to (MCVaR).

Proof. If $(\overline{\boldsymbol{x}}, \bar{y})$ is an optimal solution to (18), then due to Corollary $4.2, \overline{\boldsymbol{x}}$ is also an optimal solution to $\min _{\boldsymbol{x} \in X} \sum_{i=1}^{I} \lambda_{i} \phi_{\alpha_{i}}\left(\boldsymbol{x}, y^{*}(\boldsymbol{x})\right)$. Therefore, by the theory of mutliobjective programming, $\overline{\boldsymbol{x}}$ is also a Pareto efficient solution to (MCVaR) ([8]). Hence, the theorem is true.

The remained problem is that the condition (16) is too serious. For this, we consider the following single objective problem:

$$
\begin{aligned}
&(\mathrm{SCVaR}) \min \sum_{i=1}^{I} \lambda_{i} F_{\alpha_{i}}(\boldsymbol{x}, y), \\
& \text { s. t. } y \in R, \boldsymbol{x} \in X, \\
& 0 \leq \lambda_{i} \leq 1, \sum_{i=1}^{I} \lambda_{i}=1 .
\end{aligned}
$$

The difference between this programming with (18) is that $\left\{\lambda_{i}, i=1,2, \cdots, I\right\}$ are give in (18) while are decision variables here. The function by letting $\lambda_{i}$ be decision variables is to delete condition (16). Let

$$
\Lambda=\left\{\lambda=\left(\lambda_{1}, \lambda_{2}, \cdots, \lambda_{I}\right) \mid \lambda_{i} \in[0,1], i=1,2, \cdots, I, \sum_{i=1}^{I} \lambda_{i}=1\right\}
$$

We have the following better result.

Theorem 4.3 Suppose that $\Psi_{i}(\boldsymbol{x}, y)$ is strictly increasing in $y$. If $\left(\overline{\boldsymbol{x}}^{*}, \bar{y}^{*}, \bar{\lambda}^{*}\right)$ is an optimal solution to (SCVaR) and satisfies (13), then $\overline{\boldsymbol{x}}^{*}$ is a Pareto- $\boldsymbol{\alpha}$-CVaR efficient solution to (MCVaR). 
Proof. For $\lambda \in \Lambda$, let

$$
y^{*}(\boldsymbol{x}, \lambda)=\min \left\{y \mid \sum_{i=1}^{I} \lambda_{i}\left(1-\alpha_{i}\right)^{-1} \Psi_{i}(\boldsymbol{x}, y) \geq \sum_{i=1}^{I} \lambda_{i} \alpha_{i}\left(1-\alpha_{i}\right)^{-1}\right\} .
$$

We show that

$$
\min _{\boldsymbol{x} \in X} \sum_{i=1}^{I} \lambda_{i}^{*} \phi_{\alpha_{i}}\left(\boldsymbol{x}, y^{*}(\boldsymbol{x})\right)=\min _{\boldsymbol{x} \in X} \min _{\lambda \in \Lambda} \min _{y \in R} \sum_{i=1}^{I} \lambda_{i} F_{\alpha_{i}}(\boldsymbol{x}, y) .
$$

From (19), if $\left(\overline{\boldsymbol{x}}^{*}, \bar{y}^{*}, \bar{\lambda}^{*}\right)$ is an optimal solution to (SCVaR), we know from Theorem 4.2 that $\overline{\boldsymbol{x}}^{*}$ is a Pareto- $\alpha$-CVaR efficient solution to (MCVaR).

Therefore, in order to solve the multiobjective problem (MCVAR), we need only to solve the single objective problem (SCVAR), which can be solved clearly not too difficult.

\section{Conclusion}

This paper discusses the CVaR problem with multiple losses. We introduce the concepts of $\boldsymbol{\alpha}$-VaR and $\boldsymbol{\alpha}$-CVaR with the confidence level vector $\boldsymbol{\alpha}=$ $\left(\alpha_{1}, \alpha_{2}, \cdots, \alpha_{I}\right)$. It is shown that obtaining Pareto efficient solutions of the minimal $\boldsymbol{\alpha}$-CVaR can be transformed into solving a single objective problem under some mild conditions. This paper resolves the problem of multiobjective CVaR in theory, and provides the theory base to numerical calculate and help us to study theory and solution of multiobjective CVaR problems under others definitions of $\boldsymbol{\alpha}$-VaR furthermore.

\section{References}

1. Chen, J.-L. and Zhang, W.: CVaR and the unifying model of portfolio optimization CVaR (in Chinese), Systems Enginnering-Theory, Methodolgy, and Applications, 1(2002)68-71.

2. Rockafellar, R. T. and Uryasev, S.: Optimization of Conditional Value-at-Risk, Journal of Risk 2(2000)21-41.

3. Chernozhukov, V. and Umantsev, L.: Conditional Value-at-Risk: aspects of modeling and estimation, Empirical Economics, 26(2001) 271-292.

4. Andersson, F., Mausser, H., Rosen, D. and Uryasev, S.: Credit risk optimization with Conditional Value-at-Risk criterion, Math. Program. 89(2001)273-291.

5. Rockafellar R. T. and Uryasev, S.: Conditional Value-at-Risk for general loss distributions, Journal of Banking \& Finance 26(2002) 1443-1471.

6. Krokhmal, P., Palmquist, J. and Uryasev, S.: Portfolio optimization with Conditional Value-at-Risk objectives and constraints, Journal of Risk, 2(2002)124-129.

7. Wang J. H. and Li C. L., New method of measurement and control finance risk (in Chinese), Joural of Wuhan University of Techology, 2002, 24:2, pp. 60-63.

8. Sawragi, Y., Nakayama, H. and Tanino, T.: Theory of multiobjective optimization, Academic Press, New York (1985). 